\title{
Local Fractional Functional Method for Solving Diffusion Equations on Cantor Sets
}

\author{
Yuan Cao, ${ }^{1,2}$ Wei-Gang $\mathrm{Ma}^{3}$ and Lian-Chuan $\mathrm{Ma}^{1,2}$ \\ ${ }^{1}$ National Engineering Research Center of Rail Transportation Operation and Control System, Beijing Jiaotong University, \\ Beijing 100044, China \\ ${ }^{2}$ School of Electronic and Information Engineering, Beijing Jiaotong University, Beijing 100044, China \\ ${ }^{3}$ Faculty of Computer Science and Engineering, Xian University of Technology, Xi’An 710048, China
}

Correspondence should be addressed to Yuan Cao; yuancao112@163.com

Received 11 July 2014; Accepted 18 July 2014; Published 5 August 2014

Academic Editor: Xiao-Jun Yang

Copyright (C) 2014 Yuan Cao et al. This is an open access article distributed under the Creative Commons Attribution License, which permits unrestricted use, distribution, and reproduction in any medium, provided the original work is properly cited.

The analytical solutions for the diffusion equations on Cantor sets with the nondifferentiable terms are discussed by using the local fractional functional method, which is a coupling method for local fractional Fourier series and Laplace transform.

\section{Introduction}

The local fractional calculus $[1,2]$, as a new branch of fractional calculus, was successfully applied to describe the fractal problems from science and engineering. For example, the local fractional Fokker-Planck equation [3], the local fractional diffusion equations defined on Cantor sets $[4,5]$, the local fractional wave equation defined on Cantor sets $[6,7]$, the local fractional Korteweg-de Vries equation [8], the local fractional Schrödinger equation [9], local fractional NavierStokes equations on cantor sets [10], the local fractional Laplace equation [11], the local fractional heat-conduction equation [12-16], the local fractional differential equations arising in the fractal forest gap [17], and others [18-21] were discussed.

In this paper, we consider the local fractional diffusion equations defined on Cantor sets [5] given by

$$
u_{x x}^{2 \alpha}=\frac{1}{a^{2 \alpha}} u_{t}^{\alpha}
$$

subject to the initial-boundary conditions

$$
\begin{gathered}
\frac{\partial^{\alpha}}{\partial x^{\alpha}} u(0, t)=g(t), \quad u(0, t)=f(t), \\
u(x, 0)=u(x, l)=0,
\end{gathered}
$$

where the local fractional partial derivatives denote

$$
u_{t}^{\alpha}=\frac{\partial^{\alpha} u(x, t)}{\partial t^{\alpha}}, \quad u_{y x}^{2 \alpha}=\frac{\partial^{2 \alpha} u(x, t)}{\partial x^{\alpha} \partial y^{\alpha}}
$$

and $u(x, t), g(t)$, and $f(t)$ are the local fractional continuous functions. In the high-speed railway healthy monitor system, the problems of diffusion equations with the nondifferentiable terms always exist in fault diagnosing of high-speed trains and their control systems, so we solve this by the local fractional diffusion equations defined on Cantor sets. The local fractional function decomposition method structured in $[11,22]$, which is a coupling method of the local fractional Fourier series $[21,22]$ and the Yang-Laplace transform $[14,16$, $18,22]$, was used to solve the inhomogeneous local fractional wave equations defined on Cantor sets. The main aim of this paper is to discuss the local fractional diffusion equations defined on Cantor sets by the local fractional functional method.

The paper is organized as follows. In Section 2 the basic theory of the local fractional calculus and the Yang-Laplace transform were given. In Section 3, the local fractional functional method is analyzed. Section 4 presents the applications for the local fractional diffusion equations defined on Cantor sets. Finally, the conclusions are given in Section 5. 


\section{Preliminaries}

In this section, we present the basic theory of the local fractional calculus and the local fractional Laplace transform.

Definition 1 (see $[1,5-7]$ ). The local fractional derivative of $f(x)$ at $x=x_{0}$ is given as follows:

$$
\begin{aligned}
D_{x}^{\alpha} f\left(x_{0}\right) & =\left.\frac{d^{\alpha}}{d x^{\alpha}} f(x)\right|_{x=x_{0}}=f^{(\alpha)}(x) \\
& =\lim _{x \rightarrow x_{0}} \frac{\Delta^{\alpha}\left(f(x)-f\left(x_{0}\right)\right)}{\left(x-x_{0}\right)^{\alpha}},
\end{aligned}
$$

where $\Delta^{\alpha}\left(f(x)-f\left(x_{0}\right)\right) \cong \Gamma(\alpha+1) \Delta\left(f(x)-f\left(x_{0}\right)\right)$.

The local fractional partial derivative of order $\alpha$ is defined as follows [1]:

$$
\begin{aligned}
\left.\frac{\partial^{\alpha}}{\partial x^{\alpha}} f(x, y)\right|_{x=x_{0}} & =f^{(\alpha)}(x, y) \\
& =\lim _{x \rightarrow x_{0}} \frac{\Delta^{\alpha}\left(f(x, y)-f\left(x_{0}, y\right)\right)}{\left(x-x_{0}\right)^{\alpha}}
\end{aligned}
$$

and the local fractional partial derivative of high order [1] is

$$
\frac{\partial^{k \alpha} f(x, y)}{x^{k \alpha}}=\overbrace{\frac{\partial^{\alpha}}{\partial x^{\alpha}} \frac{\partial^{\alpha}}{\partial x^{\alpha}} \cdots \frac{\partial^{\alpha}}{\partial x^{\alpha}}}^{k \text { times }} f(x, y),
$$

where $\Delta^{\alpha}\left(f(x)-f\left(x_{0}\right)\right) \cong \Gamma(\alpha+1) \Delta\left(f(x)-f\left(x_{0}\right)\right)$.

Definition 2 (see $[1,8-12]$ ). Let us consider a partition of the interval $[a, b]$, which is denoted as $\left(t_{j}, t_{j+1}\right), j=0, \ldots, N-1$, $t_{0}=a$ and $t_{N}=b$ with $\Delta t_{j}=t_{j+1}-t_{j}$ and $\Delta t=$ $\max \left\{\Delta t_{0}, \Delta t_{1}, \ldots\right\}$. Local fractional integral of $f(x)$ in the interval $[a, b]$ is defined as follows:

$$
\begin{aligned}
{ }_{a} I_{b}^{(\alpha)} f(x) & =\frac{1}{\Gamma(1+\alpha)} \int_{a}^{b} f(t)(d t)^{\alpha} \\
& =\frac{1}{\Gamma(1+\alpha)} \lim _{\Delta t \rightarrow 0} \sum_{j=0}^{N-1} f\left(t_{j}\right)\left(\Delta t_{j}\right)^{\alpha} .
\end{aligned}
$$

Definition 3 (see $[1,5,11,16,21])$. The Mittag Leffler, sine and cosine functions defined on Cantor sets are given as follows:

$$
\begin{aligned}
& E_{\alpha}\left(x^{\alpha}\right)=\sum_{k=0}^{\infty} \frac{x^{\alpha k}}{\Gamma(1+k \alpha)}, \\
& \sin _{\alpha} x^{\alpha}=\sum_{k=0}^{\infty}(-1)^{k} \frac{x^{\alpha(2 k+1)}}{\Gamma[1+\alpha(2 k+1)]}, \\
& \cos _{\alpha} x^{\alpha}=\sum_{k=0}^{\infty}(-1)^{k} \frac{x^{2 \alpha k}}{\Gamma(1+2 \alpha k)},
\end{aligned}
$$

for $x \in R, 0<\alpha<1$.
Definition 4 (see $[11,20-22]$ ). Let $f(x)$ be $2 l$-periodic. For $k \epsilon$ $Z$, local fraction Fourier series of $f(x)$ is given as

$$
f(x)=\frac{a_{0}}{2}+\sum_{k=1}^{\infty}\left(a_{n} \cos _{\alpha} \frac{\pi^{\alpha}(k x)^{\alpha}}{l^{\alpha}}+b_{n} \sin _{\alpha} \frac{\pi^{\alpha}(k x)^{\alpha}}{l^{\alpha}}\right),
$$

where the local fraction Fourier coefficients are as follows:

$$
\begin{aligned}
& a_{n}=\frac{2}{l^{\alpha}} \int_{0}^{l} f(x) \cos _{\alpha} \frac{\pi^{\alpha}(k x)^{\alpha}}{l^{\alpha}}(d x)^{\alpha}, \\
& b_{n}=\frac{2}{l^{\alpha}} \int_{0}^{l} f(x) \sin _{\alpha} \frac{\pi^{\alpha}(k x)^{\alpha}}{l^{\alpha}}(d x)^{\alpha} .
\end{aligned}
$$

Definition 5 (see $[14,16,18,22])$. Let $(1 / \Gamma(1+$ a)) $\int_{0}^{\infty}|f(x)|(d x)^{\alpha}<k<\infty$. The local fractional Laplace transform of $f(x)$ is given as

$$
\begin{array}{r}
\widetilde{L}_{\alpha}\{f(x)\}=f_{s}^{\widetilde{L}, \alpha}(s)=\frac{1}{\Gamma(1+\alpha)} \int_{0}^{\infty} E_{\alpha}\left(-s^{\alpha} x^{\alpha}\right) f(x)(d x)^{\alpha}, \\
0<\alpha \leq 1 .
\end{array}
$$

The inverse formula local fractional Laplace transform of $f(x)$ is given as $[14,16,18,22]$

$$
\begin{aligned}
f(x) & =\widetilde{L}_{\alpha}^{-1}\left\{f_{s}^{L, \alpha}(s)\right\} \\
& =\frac{1}{(2 \pi)^{\alpha}} \int_{\beta-i \infty}^{\beta+i \infty} E_{\alpha}\left(s^{\alpha} x^{\alpha}\right) f_{s}^{\widetilde{L}, \alpha}(s)(d s)^{\alpha},
\end{aligned}
$$

where $f(x)$ is local fractional continuous, $s^{\alpha}=\beta^{\alpha}+i^{\alpha} \infty^{\alpha}$ and $\operatorname{Re}(s)=\beta>0$.

There is the following formula $[14,16,18,22]$ :

$$
\widetilde{L}_{\alpha}\left\{y^{(2 \alpha)}(x)\right\}=s^{2 \alpha} \widetilde{L}_{\alpha}\{y(x)\}-s^{\alpha} y(0)-f^{(\alpha)}(0) .
$$

The basic properties of the local fractional calculus and the local fractional Laplace transform were listed in $[1,14,16,18$, 22].

\section{Analysis of the Local Fractional Functional Method}

In this section, we introduce the local fractional functional method for the local fractional diffusion equations defined on Cantor sets [11, 22].

Let us consider the nondifferentiable decomposition of the function with the nondifferentiable systems $\left\{\sin _{\alpha} n^{\alpha}(\pi t / l)^{\alpha}\right\}$. There are the following functional coefficients of (1) and (2), which are given as follows:

$$
\begin{gathered}
u(x, t)=\sum_{n=1}^{\infty} A_{n}(x) \sin _{\alpha} n^{\alpha}\left(\frac{\pi t}{l}\right)^{\alpha}, \\
g(t)=\sum_{n=1}^{\infty} C_{n} \sin _{\alpha} n^{\alpha}\left(\frac{\pi t}{l}\right)^{\alpha}, \\
f(t)=\sum_{n=1}^{\infty} D_{n} \sin _{\alpha} n^{\alpha}\left(\frac{\pi t}{l}\right)^{\alpha},
\end{gathered}
$$


where

$$
\begin{aligned}
A_{n}(x) & =\frac{2}{l^{\alpha}} \int_{0}^{1} u(x, t) \sin _{\alpha} n^{\alpha}\left(\frac{\pi t}{l}\right)^{\alpha}(d t)^{\alpha}, \\
C_{n} & =\frac{2}{l^{\alpha}} \int_{0}^{1} g(t) \sin _{\alpha} n^{\alpha}\left(\frac{\pi t}{l}\right)^{\alpha}(d t)^{\alpha}, \\
D_{n} & =\frac{2}{l^{\alpha}} \int_{0}^{1} f(t) \sin _{\alpha} n^{\alpha}\left(\frac{\pi t}{l}\right)^{\alpha}(d t)^{\alpha} .
\end{aligned}
$$

If we submit (14) into (1) and (2), then we have

$$
\begin{gathered}
\frac{d^{2 \alpha}}{d x^{2 \alpha}} A_{n}(x)=\frac{1}{a^{2 \alpha}} A_{n}(x)\left(\frac{n \pi}{l}\right)^{\alpha}, \\
A_{n}^{(\alpha)}(0)=C_{n}, \\
A_{n}(0)=D_{n} .
\end{gathered}
$$

Taking the local fractional Laplace transform of (16) gives

$$
\widetilde{L}_{\alpha}\left\{\frac{d^{2 \alpha}}{d x^{2 \alpha}} A_{n}(x)\right\}=s^{2 \alpha} \widetilde{L}_{\alpha}\left\{A_{n}(x)\right\}-s^{\alpha} A_{n}(0)-A_{n}^{(\alpha)}(0),
$$

which leads to

$$
s^{2 \alpha} \widetilde{L}_{\alpha}\left\{A_{n}(x)\right\}-s^{\alpha} D_{n}-C_{n}=\frac{1}{a^{2 \alpha}}\left(\frac{n \pi}{l}\right)^{\alpha} \widetilde{L}_{\alpha}\left\{A_{n}(x)\right\} .
$$

We can rewrite (18) as

$$
\begin{aligned}
A_{n}(s) \\
=\widetilde{L}_{\alpha}\left\{A_{n}(x)\right\} \\
=\frac{s^{\alpha} D_{n}+C_{n}}{s^{2 \alpha}-\left(1 / a^{2 \alpha}\right)(n \pi / l)^{\alpha}} \\
=\frac{D_{n}}{s^{\alpha}-\left(1 / a^{\alpha}\right)(n \pi / l)^{\alpha / 2}}+\frac{\left(C_{n}-\left(1 / a^{\alpha}\right)(n \pi / l)^{\alpha / 2} D_{n}\right)}{s^{2 \alpha}-\left(1 / a^{2 \alpha}\right)(n \pi / l)^{\alpha}} \\
=\frac{D_{n}}{s^{\alpha}-\left(1 / a^{\alpha}\right)(n \pi / l)^{\alpha / 2}} \\
\quad+P\left(\frac{1}{s^{\alpha}-\left(1 / a^{\alpha}\right)(n \pi / l)^{\alpha / 2}}-\frac{1}{s^{\alpha}+\left(1 / a^{\alpha}\right)(n \pi / l)^{\alpha / 2}}\right),
\end{aligned}
$$

where

$$
P=\frac{C_{n}-\left(1 / a^{\alpha}\right)(n \pi / l)^{\alpha / 2} D_{n}}{\left(1 / a^{\alpha}\right)(n \pi / l)^{\alpha / 2}} .
$$

The inverse formula local fractional Laplace transform of (19) gives

$$
\begin{aligned}
A_{n}(x) & \\
= & \widetilde{L}_{\alpha}^{-1}\left\{A_{n}(s)\right\} \\
= & \frac{1}{(2 \pi)^{\alpha}} \int_{\beta-i \infty}^{\beta+i \infty} E_{\alpha}\left(s^{\alpha} x^{\alpha}\right) A_{n}(s)(d s)^{\alpha} \\
= & \frac{1}{(2 \pi)^{\alpha}} \\
& \times \int_{\beta-i \infty}^{\beta+i \infty} E_{\alpha}\left(s^{\alpha} x^{\alpha}\right)\left\{\frac{D_{n}}{s^{\alpha}-\left(1 / a^{\alpha}\right)(n \pi / l)^{\alpha / 2}}\right. \\
& +P\left(\frac{1}{s^{\alpha}-\left(1 / a^{\alpha}\right)(n \pi / l)^{\alpha / 2}}\right. \\
= & D_{n} E_{\alpha}\left(\frac{1}{a^{\alpha}}\left(\frac{n \pi}{l}\right)^{\alpha / 2} x^{\alpha}\right)+P E_{\alpha}\left(\frac{1}{a^{\alpha}}\left(\frac{n \pi}{l}\right)^{\alpha / 2} x^{\alpha}\right) \\
& -P E_{\alpha}\left(-\frac{1}{a^{\alpha}}\left(\frac{n \pi}{l}\right)^{\alpha / 2} x^{\alpha}\right),
\end{aligned}
$$

where

$$
P=\frac{C_{n}-\left(1 / a^{\alpha}\right)(n \pi / l)^{\alpha / 2} D_{n}}{\left(1 / a^{\alpha}\right)(n \pi / l)^{\alpha / 2}} .
$$

Hence, the solution of (1) reads as follows:

$$
\begin{aligned}
u & (x, t) \\
= & \sum_{n=1}^{\infty} A_{n}(x) \sin _{\alpha} n^{\alpha}\left(\frac{\pi t}{l}\right)^{\alpha} \\
= & \sum_{n=1}^{\infty} D_{n} E_{\alpha}\left(\frac{1}{a^{\alpha}}\left(\frac{n \pi}{l}\right)^{\alpha / 2} x^{\alpha}\right) \sin _{\alpha} n^{\alpha}\left(\frac{\pi t}{l}\right)^{\alpha} \\
& +\sum_{n=1}^{\infty} P E_{\alpha}\left(\frac{1}{a^{\alpha}}\left(\frac{n \pi}{l}\right)^{\alpha / 2} x^{\alpha}\right) \sin _{\alpha} n^{\alpha}\left(\frac{\pi t}{l}\right)^{\alpha} \\
& -\sum_{n=1}^{\infty} P E_{\alpha}\left(-\frac{1}{a^{\alpha}}\left(\frac{n \pi}{l}\right)^{\alpha / 2} x^{\alpha}\right) \sin _{\alpha} n^{\alpha}\left(\frac{\pi t}{l}\right)^{\alpha},
\end{aligned}
$$

where

$$
P=\frac{C_{n}-\left(1 / a^{\alpha}\right)(n \pi / l)^{\alpha / 2} D_{n}}{\left(1 / a^{\alpha}\right)(n \pi / l)^{\alpha / 2}} .
$$

\section{The Exact Solutions for Local Fractional Diffusion Equations Defined on Cantor Sets}

In this section we give two examples for initial boundary problems for local fractional diffusion equations defined on Cantor sets. 
Example 6. The initial-boundary values of (1) read as follows:

$$
\begin{gathered}
\frac{\partial^{\alpha}}{\partial x^{\alpha}} u(0, t)=\sin \left(t^{\alpha}\right), \quad u(0, t)=0, \\
u(x, 0)=u(x, \pi)=0 .
\end{gathered}
$$

Making use of (14), we obtain the following formulas:

$$
\begin{gathered}
\sin \left(t^{\alpha}\right)=\sum_{n=1}^{\infty} C_{n} \sin _{\alpha} n^{\alpha} t^{\alpha}, \\
0=\sum_{n=1}^{\infty} D_{n} \sin _{\alpha} n^{\alpha} t^{\alpha}
\end{gathered}
$$

which lead to the following parameters:

$$
\begin{array}{ll}
C_{n}=1, & n=1, \\
C_{n}=0, & n>1, \\
D_{n}=0, & n \geq 1 .
\end{array}
$$

Therefore, (23) gives the nondifferentiable solution of (1) with initial-boundary values (25)

$$
\begin{aligned}
u(x, t) & \\
= & \sum_{n=1}^{\infty} A_{n}(x) \sin _{\alpha} n^{\alpha} t^{\alpha} \\
= & \sum_{n=1}^{\infty} D_{n} E_{\alpha}\left(\frac{1}{a^{\alpha}} x^{\alpha}\right) \sin _{\alpha} n^{\alpha} t^{\alpha}+\sum_{n=1}^{\infty} P E_{\alpha}\left(\frac{1}{a^{\alpha}} x^{\alpha}\right) \sin _{\alpha} n^{\alpha} t^{\alpha} \\
& -\sum_{n=1}^{\infty} P E_{\alpha}\left(-\frac{1}{a^{\alpha}} x^{\alpha}\right) \sin _{\alpha} n^{\alpha} t^{\alpha} \\
= & a^{\alpha} E_{\alpha}\left(\frac{1}{a^{\alpha}} x^{\alpha}\right) \sin _{\alpha} t^{\alpha}-a^{\alpha} E_{\alpha}\left(-\frac{1}{a^{\alpha}} x^{\alpha}\right) \sin _{\alpha} t^{\alpha} .
\end{aligned}
$$

When $a=1$, we get the nondifferentiable solution

$$
u(x, t)=E_{\alpha}\left(x^{\alpha}\right) \sin _{\alpha} t^{\alpha}-E_{\alpha}\left(-x^{\alpha}\right) \sin _{\alpha} t^{\alpha},
$$

and its graph is shown in Figure 1.

Example 7. We present the initial-boundary values of (1) as

$$
\begin{gathered}
\frac{\partial^{\alpha}}{\partial x^{\alpha}} u(0, t)=\sin \left(t^{\alpha}\right), \quad u(0, t)=\sin \left(t^{\alpha}\right), \\
u(x, 0)=u(x, \pi)=0 .
\end{gathered}
$$

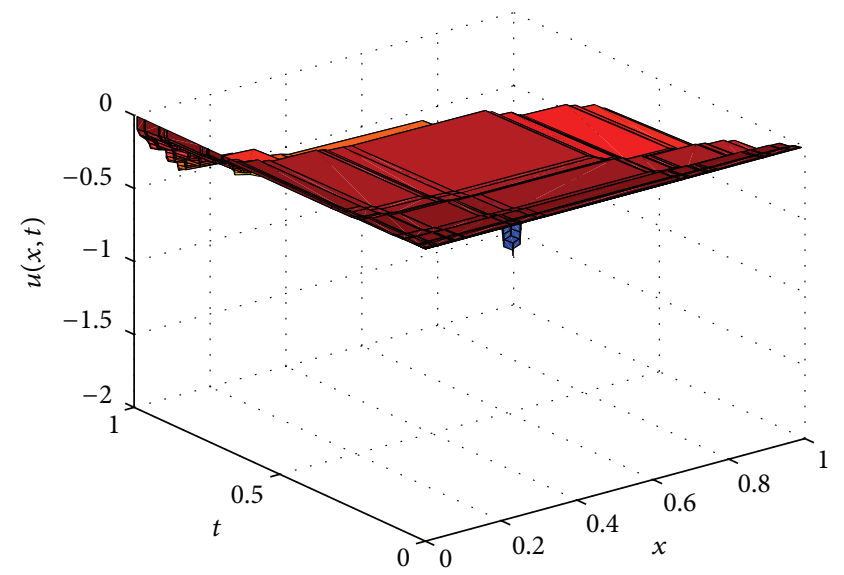

FIgURE 1: The solution of (1) with initial-boundary value (25) when $a=1$ and $\alpha=\ln 2 / \ln 3$.

Using the relation (14), we get

$$
\begin{gathered}
0=\sum_{n=1}^{\infty} C_{n} \sin _{\alpha} n^{\alpha} t^{\alpha}, \\
\sin \left(t^{\alpha}\right)=\sum_{n=1}^{\infty} D_{n} \sin _{\alpha} n^{\alpha} t^{\alpha}
\end{gathered}
$$

which reduce to

$$
\begin{array}{ll}
C_{n}=0, & n \geq 1, \\
D_{n}=1, & n=1, \\
D_{n}=0, & n>1 .
\end{array}
$$

Using (23), we hence have the nondifferentiable solution of (1) with initial-boundary values (30), which is given as

$$
\begin{aligned}
u(x, t) & \\
= & \sum_{n=1}^{\infty} A_{n}(x) \sin _{\alpha} n^{\alpha} t^{\alpha} \\
= & \sum_{n=1}^{\infty} D_{n} E_{\alpha}\left(\frac{1}{a^{\alpha}} x^{\alpha}\right) \sin _{\alpha} n^{\alpha} t^{\alpha}+\sum_{n=1}^{\infty} P E_{\alpha}\left(\frac{1}{a^{\alpha}} x^{\alpha}\right) \sin _{\alpha} n^{\alpha} t^{\alpha} \\
& -\sum_{n=1}^{\infty} P E_{\alpha}\left(-\frac{1}{a^{\alpha}} x^{\alpha}\right) \sin _{\alpha} n^{\alpha} t^{\alpha} \\
= & E_{\alpha}\left(\frac{1}{a^{\alpha}} x^{\alpha}\right) \sin _{\alpha} t^{\alpha}-a^{\alpha} E_{\alpha}\left(\frac{1}{a^{\alpha}} x^{\alpha}\right) \sin _{\alpha} t^{\alpha} \\
& +a^{\alpha} E_{\alpha}\left(-\frac{1}{a^{\alpha}} x^{\alpha}\right) \sin _{\alpha} t^{\alpha} .
\end{aligned}
$$

For $a=1$, the nondifferentiable solution rewrites as follows:

$$
u(x, t)=E_{\alpha}\left(-x^{\alpha}\right) \sin _{\alpha} t^{\alpha},
$$

and its graph is illustrated in Figure 2. 


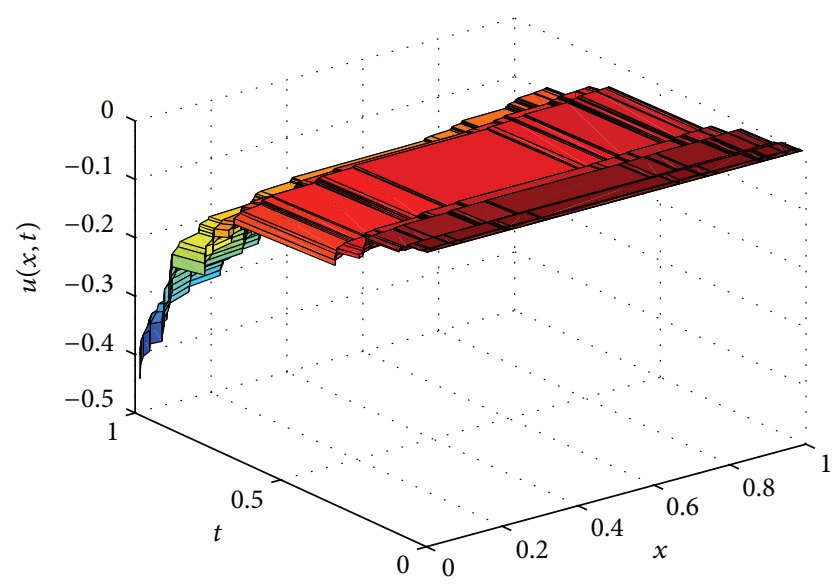

FIgURE 2: The solution of (1) with initial-boundary value (30) when $a=1$ and $\alpha=\ln 2 / \ln 3$.

\section{Conclusions}

Local fractional calculus was applied to describe the physical problems because of nondifferentiable characteristics. In this work, the initial-boundary value problems for the diffusion equation on Cantor sets within the local fractional derivatives were investigated by using the local fractional functional method, which is a coupling method for local fractional Fourier series and Laplace transform based upon the nondifferentiable decomposition of the function with the nondifferentiable systems. The two examples are given to express the efficiency of the presented method and their graphs are also obtained. The results of this paper could provide the theory support to the problems diffusion equations with the nondifferentiable terms in health monitor of highspeed trains and their control systems.

\section{Conflict of Interests}

The authors declare that there is no conflict of interests regarding the publication of this paper.

\section{References}

[1] X. J. Yang, Advanced Local Fractional $C$ alculus and $I$ ts $A$ pplications, World Science, New York, NY, USA, 2012.

[2] A. Babakhani and V. D. Gejji, "On calculus of local fractional derivatives," Journal of Mathematical Analysis and Applications, vol. 270, no. 1, pp. 66-79, 2002.

[3] K. M. Kolwankar and A. D. Gangal, "Local fractional FokkerPlanck equation," Physical Review Letters, vol. 80, no. 2, pp. 214217, 1998.

[4] A. Carpinteri and A. Sapora, "Diffusion problems in fractal media defined on Cantor sets," ZAMM Zeitschrift fur Angewandte Mathematik und Mechanik, vol. 90, no. 3, pp. 203-210, 2010.

[5] X. Yang, D. Baleanu, and W. P. Zhong, "Approximate solutions for diffusion equations on Cantor space-time," Proceedings of the Romanian Academy, Series A, vol. 14, no. 2, pp. 127-133, 2013.
[6] J. Ahmad, S. T. Mohyud-Din, and X. J. Yang, "Local fractional decomposition method on wave equation in fractal strings," Mitteilungen Klosterneuburg, vol. 64, no. 2, pp. 98-105, 2014.

[7] X. J. Yang, H. M. Srivastava, J. H. He, and D. Baleanu, "Cantortype cylindrical-coordinate method for differential equations with local fractional derivatives," Physics Letters A, vol. 377, no. 28-30, pp. 1696-1700, 2013.

[8] X. J. Yang, J. Hristov, and H. M. Srivastava, "Modelling fractal waves on shallow water surfaces via local fractional Kortewegde Vries equation," Abstract and Applied Analysis, vol. 2014, Article ID 278672, 10 pages, 2014.

[9] X. J. Yang, D. Baleanu, and J. A. T. Machado, "Mathematical aspects of the Heisenberg uncertainty principle within local fractional Fourier analysis," Boundary Value Problems, vol. 2013, no. 1, pp. 1-16, 2013.

[10] X. Yang, D. Baleanu, and J. A. Tenreiro Machado, "Systems of navier-stokes equations on cantor sets," Mathematical Problems in Engineering, vol. 2013, Article ID 769724, 8 pages, 2013.

[11] S. Yan, H. Jafari, and H. K. Jassim, "Local fractional Adomain d ecomposition and function decomposition methods for Laplace equation within local fractional operators," Advances in Mathematical Physics, vol. 2014, Article ID 161580, 7 pages, 2014.

[12] A. M. Yang, Y. Z. Zhang, and X. L. Zhang, "The nondifferentiable solution for local fractional Tricomi equation arising in fractal transonic flow by local fractional variational iteration method," Advances in Mathematical Physics, vol. 2014, 6 pages, 2014.

[13] A. Yang, C. Zhang, H. Jafari, C. Cattani, and Y. Jiao, "Picard successive approximation method for solving differential equations arising in fractal heat transfer with local fractional derivative," Abstract and Applied Analysis, vol. 2014, Article ID 395710, 5 pages, 2014.

[14] Y. Z. Zhang, A. M. Yang, and Y. Long, "Initial boundary value problem for fractal heat equation in the semi-infinite region by Yang-Laplace transform," Thermal Science, vol. 18, no. 2, pp. 677-681, 2014.

[15] J. H. He and F. J. Liu, "Local fractional variational iteration method for fractal heat transfer in silk cocoon hierarchy," Nonlinear Science Letters A, vol. 4, no. 1, pp. 15-20, 2013.

[16] C. F. Liu, S. S. Kong, and S. J. Yuan, "Reconstructive schemes for variational iteration method within Yang-Laplace transform with application to fractal heat conduction problem," Thermal Science, vol. 17, no. 3, pp. 715-721, 2013.

[17] C. Long, Y. Zhao, and H. Jafari, "Mathematical models arising in the fractal forest gap via local fractional calculus," Abstract and Applied Analysis, vol. 2014, Article ID 782393, 6 pages, 2014.

[18] C. G. Zhao, A. M. Yang, H. Jafari, and A. Haghbin, “The YangLaplace transform for solving the IVPs with local fractional derivative," Abstract and Applied Analysis, vol. 2014, Article ID 386459, 5 pages, 2014.

[19] J. H. He, "Exp-function method for fractional differential equations," International Journal of Nonlinear Sciences and Numerical Simulation, vol. 14, no. 6, pp. 363-366, 2013.

[20] Z. Chen, C. Cattani, and W. P. Zhong, "Signal processing for non-differentiable data defined on Cantor sets: a local fractional fourier series approach," Advances in Mathematical Physics, vol. 2014, Article ID 561434, 7 pages, 2014. 
[21] G. A. Anastassiou and O. Duman, Advances in Applied Mathematics and Approximation Theory, Springer, New York, NY, USA, 2013.

[22] S. Wang, Y. Yang, and H. K. Jassim, "Local fractional function decomposition method for solving inhomogeneous wave equations with local fractional derivative," Abstract and Applied Analysis, vol. 2014, Article ID 176395, 7 pages, 2014. 


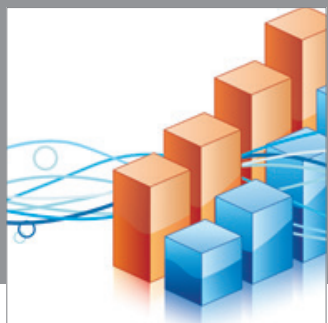

Advances in

Operations Research

mansans

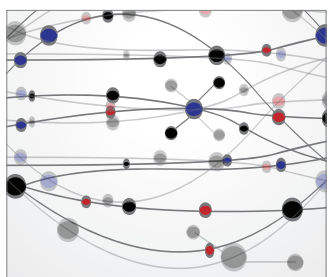

The Scientific World Journal
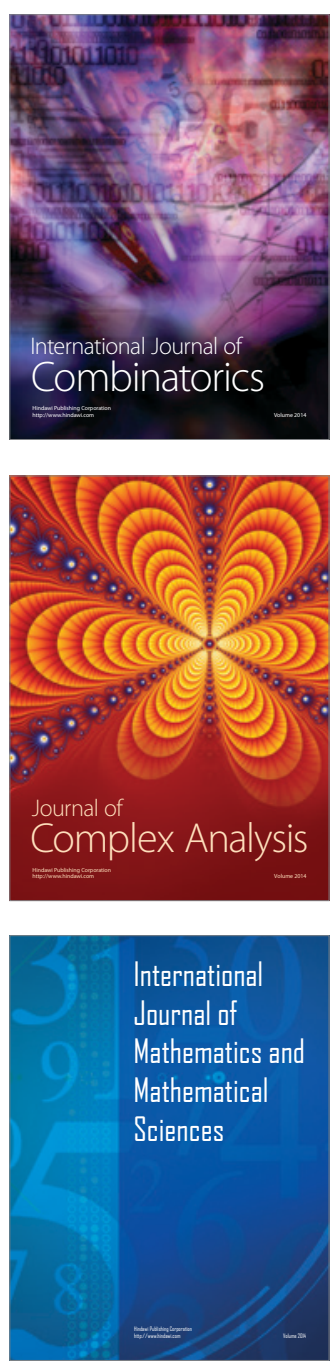
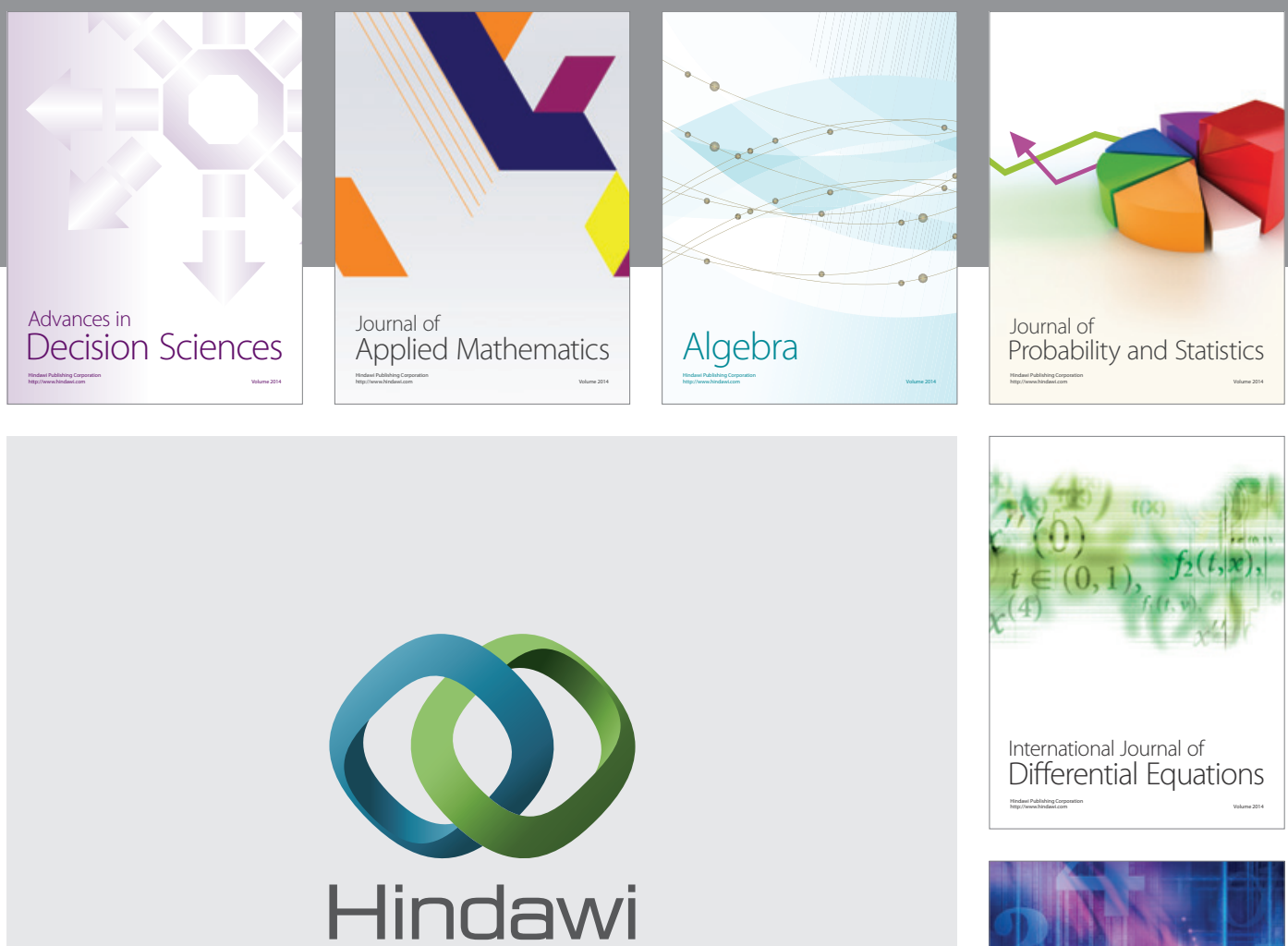

Submit your manuscripts at http://www.hindawi.com
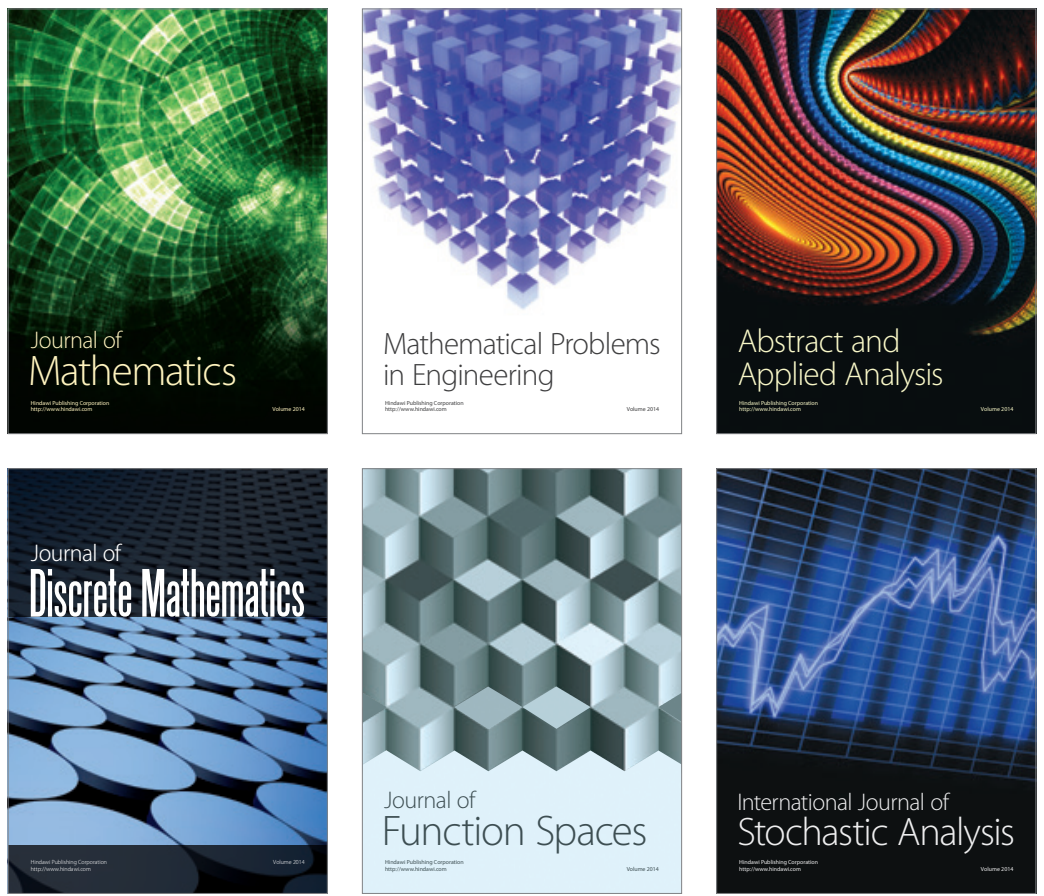

Journal of

Function Spaces

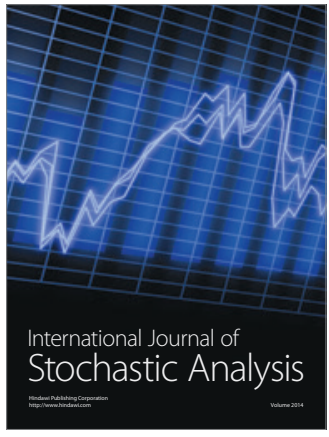

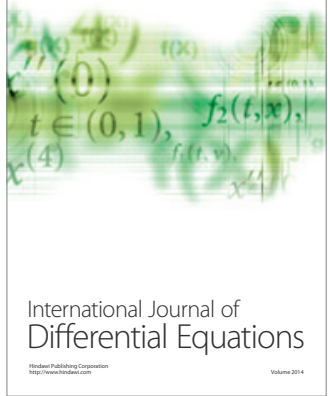
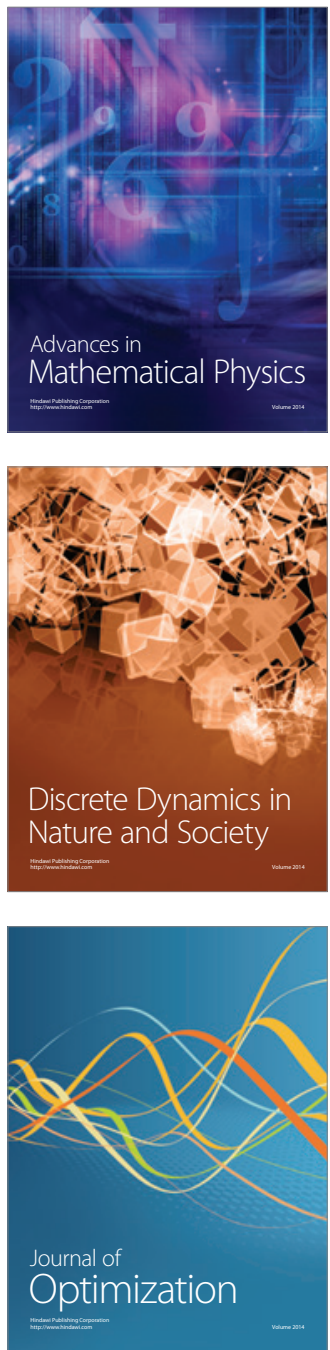